\title{
NATURALEZA Y TRASCENDENCIA EN EL ARTE CONTEMPORÁNEO. CHRIS DRURY*
}

\author{
Macarena Moreno Moreno \\ Universidad Rey Juan Carlos de Madrid \\ narecama13@yahoo.es
}

\section{RESUMEN}

La expresión de la trascendencia en el arte ha sido llevada a cabo por muchos artistas en su obra. Hay creadores que esa expresión trascendente la realizan en la naturaleza o buscando su inspiración y experiencia numinosa en ella. Aunque la conexión entre estos tres conceptos de arte, naturaleza y trascendencia ha merecido en su momento la atención académica necesaria, en las últimas décadas ha quedado en un segundo plano. En el presente artículo queremos dar visibilidad a los artistas contemporáneos que enlazan estos tres conceptos en su obra, artistas que con la contemplación de la naturaleza acceden a una percepción más profunda, a sentir la realidad como parte de sí mismos. Examinamos esta manera de crear a través de la obra de un artista anglosajón: Chris Drury. Recorrer sus principales etapas creativas nos va a permitir apreciar sus aportaciones y plantear una reflexión en torno a la intencionalidad y actitud del artista en el acto de crear y cómo este significado es transmitido al espectador, apoyando nuestro estudio en las herramientas existentes en sus creaciones, siempre observadas desde el punto de vista del propio artista.

PAlabras Clave: arte contemporáneo, efímero, naturaleza, creación, espiritual.

\section{NATURE AND TRANSCENDENCE IN CONTEMPORARY ART. CHRIS DRURY}

\section{Abstract}

The expression of transcendence in art has been carried out by many artists in their artwork. There are creators that perform that transcendent expression in nature or looking for inspiration and numinous experience in it. So far, the combination of these three concepts: Art, Nature and Transcendence deserved the necessary academic attention at the time, in recent decades, it has been in the background. Through this article we aim to give visibility to contemporary artists who link these three concepts in their work. Artists who have reached a deeper perception through the contemplation of nature, feeling reality as part of themselves. We examine this way of creating through the artwork of an Anglo-Saxon artist: Chris Drury. Going through his main creative stages will allow us to appreciate his contributions and develop a reflection on the intentionality and attitude of the artist in the act of creating and how this meaning is transmitted to the viewer, supporting our study in the existing tools of his creations, always considered from the point of view of the artist himself. We intend to contribute in this way to give visibility to active artists who, like him, harmonize these three concepts.

KEYWORDs: contemporary art, ephemeral, nature, creation, spiritual. 


\section{INTRODUCCIÓN}

La naturaleza es fuente y nutriente de vida, en ella se condensan los misterios que armonizan el orden y el caos del universo. A lo largo de toda la historia del arte, podemos encontrar ejemplos en los que aparecen creadores que muestran en su expresión artística su relación con la naturaleza y la búsqueda de lo sublime, como es el caso de Caspar David Friedrich ${ }^{1}$. Pero nuestra aportación está centrada en autores contemporáneos, entre los que podríamos mencionar a Giuseppe Penone, enmarcado en el movimiento Arte Povera y conocido por sus enormes esculturas de árboles, en las que indaga entre el vínculo del ser humano y el mundo natural; Alfio Bonanno, que utiliza los propios materiales que la naturaleza le ofrece para crear sus obras y que considera que la felicidad se da cuando el vínculo entre arte y naturaleza no se rompe ${ }^{2}$; Christiane Löhr, Nils Udo, Nikolaus Lang y otros muchos artistas europeos. En este artículo queremos acercarnos al artista Chris Drury. Este acercamiento lo hacemos fundamentalmente desde el punto de vista del artista, para llegar más a fondo a las intenciones del creador y no tanto a una clasificación técnica o histórica. Indudablemente mencionaremos datos necesarios para la comprensión del marco biográfico-creativo, pero no queremos convertir esta aportación en una lista de fechas y exhibiciones que fácilmente se pueden consultar en su biografía.

Como punto de partida veremos los aspectos coincidentes con la obra de Andy Goldsworthy y otros artistas coetáneos y también aquello que los separa y hace diferentes de otros creadores que trabajan dentro de la corriente de Land Art. El artículo lo centramos fundamentalmente en la obra de Chris Drury, ya que su obra es menos conocida fuera de Reino Unido. Seguimos un orden de exposición basado en el análisis de sus principales etapas creativas y sus obras más significativas, llegando finalmente a concluir con sus principales reflexiones y aportaciones.

\section{ASPECTOS COINCIDENTES Y DISCORDANTES}

Caspar David Friedrich, pintor paisajista del romanticismo alemán del s. XIX, afirmaba: «Debo rendirme a lo que me rodea, unirme con las nubes y con las piedras, para ser lo que soy. Necesito soledad para entrar en comunicación con

* Este artículo parte de una investigación realizada dentro del equipo de investigación «Innovación y análisis de la imagen», de la Facultad de Comunicación, Bellas Artes y Diseño de la Universidad Francisco de Vitoria de Madrid. Dicho proyecto está financiado por la Comunidad de Madrid y por el Fondo Social Europeo. En el congreso V International Conference on Mythcriticism: Myth and Audiovisual Creation, celebrado en la Universidad Francisco de Vitoria de Madrid del 15 al 26 de octubre de 2018, ya expusimos una ponencia relacionada con el tema: "Chris Drury y Andy Goldsworthy. Poetas del silencio, lo efímero y la transformación».

${ }^{1}$ María Eugenia Manrique, Arte, Naturaleza y espiritualidad (Barcelona: Kairós, 2018), 19.

${ }^{2}$ Michael Lailach, Land art (Colonia: Taschen, 2007), 47. 
la naturaleza ${ }^{3}$. Con estas palabras desvelaba el camino que como artista había elegido para su evolución espiritual, como ser humano en armonía con la naturaleza. Esta relación mística y mítica con el entorno volvemos a encontrarla dos siglos después en algunos artistas actuales.

Chris Drury forma parte de una nueva generación de artistas como Andy Goldsworthy, Hamilton Fulton o Richard Long, entre otros, adscritos todavía a la corriente artística del Land Art. Sin embargo, ni él ni Goldsworthy participaron en las históricas exposiciones de los años sesenta. Su producción es una obra artística circunstancial y no obedece a ningún programa o manifiesto estético. Ambos comparten una reflexión en torno al espacio y el tiempo, su carácter es efímero y se localiza generalmente en paisajes inhóspitos. Su propuesta artística indaga en el ser humano y su lado más trascendente. Nos acercamos a ellos desde el punto de vista del artista creador, su punto de partida, su proceso y su obra.

Dentro de las tendencias que tiene el Land Art, Drury y Goldsworthy tienen una relación más personal y mística con el entorno, emplean sus manos y lo que encuentran allí mismo. No suelen precisar de un gran equipo instrumental, salvo cuando realizan esculturas grandes. El trabajo que llevan a cabo es a menudo duro y agotador, otras veces minucioso y monótono. No realizan acciones o performances ni tampoco su obra propone proyectos megalómanos ${ }^{4}$ como es el caso de otros artistas, como Carl André o Richard Long, cuyas obras en ocasiones parecen no tener límites 5 . Una vez más, como sostiene Anna Talens en su tesis ${ }^{6}$, nos encontramos ante artistas que convierten la experiencia de la naturaleza en arte, que dialogan con ella de otra manera, que han pasado más desapercibidos, eclipsados ante las obras más llamativas y publicitadas de los artistas norteamericanos. Ellos no introducen materiales atípicos, trabajan con los elementos del lugar. Su interés en puntos geográficos específicos de la tierra, la historia de esos lugares y la relación entre el material orgánico y la presencia humana los ha apartado de aquellos que trabajan con la tierra como mero lienzo o material. Siendo este aspecto una seña de identidad.

"Como en la mayoría de los artistas de Land Art la obra es un proceso interminable donde se combina todo: el lugar, la situación, el estado atmosférico, el artista y todo lo que allí sucede» 7 . La obra transciende su carácter objetual. No se trata de un espacio representado sino experimentado. Es un escenario en el que acontece un instante. El artista no obra con una idea premeditada sobre los emplazamientos, sino que debe descubrir el paraje, escucharlo y sacar a la luz lo que está oculto

${ }^{3}$ Manrique, Arte, Naturaleza y espiritualidad, op. cit., 13.

4 William Malpas, The Srt of Andy Goldsworthy (Maidstone, Kent: Crescent Moon Publishing, 2016), 53.

${ }^{5}$ Véanse las reflexiones de Javier Maderuelo con respecto a la obra de Richad Long «A line in the Himalayas» de 1975. Maderuelo, J. Conferencia Fundación Juan March (2005), disponible en www.march.es/conferencias.

${ }^{6}$ Anna Talens Pardo, La transformación de la experiencia de la naturaleza en arte. El nomadismo y lo efimero. Tesis doctoral (Universitat Politècnica de València, 2011), 4.

7 Tonia Raquejo, Land Art (San Sebastián: Nerea, 1998), 9. 

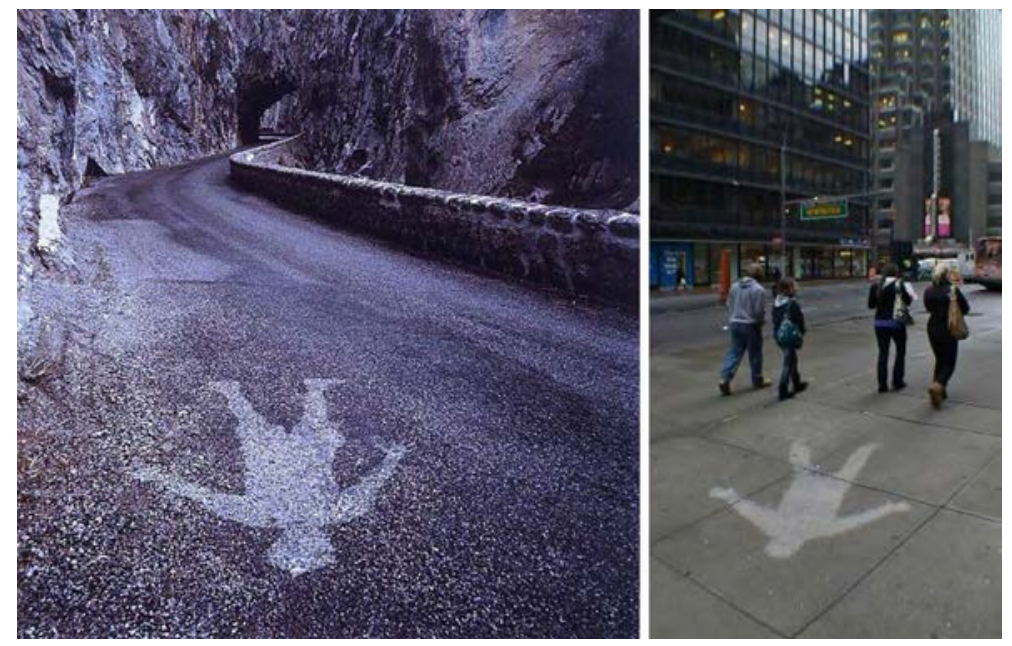

Figura 1. Sombra de lluvia, Tickon, 1993, y Rain Shadow, Times Square, 2010. (CAndy Goldsworthy.

en él. Tanto para Goldsworthy como para Drury, cada espacio requiere una intervención única, de modo que lo que construyen o modifican no podrían hacerlo en ningún otro sitio. Lo efímero sigue siendo uno de sus fundamentos, como podemos ver en muchas obras de Goldsworthy.

La serie «Huellas de lluvia» (figura 1), creada por Goldsworthy a lo largo de una década, es un buen ejemplo de ello. En un día gris se le ocurrió tumbarse en la carretera y allí con la cámara fotográfica que llevaba preparada, esperó a que la lluvia cayera. Cuando la lluvia cesó, se levantó y lo que quedó sobre el asfalto fue su silueta seca: Sombra de lluvia (1993), dejando su huella efímera en el paisaje. En Times Square (2010) quiso repetir la experiencia en un entorno más urbano, para resaltar el hecho de que los seres humanos, aunque vivan en la ciudad, siguen relacionándose con la naturaleza y dejan su huella en los lugares en los que habitan y trabajan ${ }^{8}$.

Estos artistas viven en primera persona lo que acontece en la naturaleza y su obra parte de esta experiencia exterior-interior, como veremos en el análisis de la obra de Drury. Es una obra que tiene una clara influencia del pensamiento oriental y que plantea el retorno al contacto directo con el entorno natural, a sentir su energía fortaleciendo el vínculo con el ser humano y su parte más espiritual o trascendente. Tal como explica Manrique, "para la filosofía taoísta, el arte ha de establecer un vínculo fundamental entre el ser humano y la naturaleza. Vínculo a través del

${ }^{8}$ Mel Gooding, Song of the earth. European artist and the landscape (Scotland: Thames \& Hudson, 2017), 54-57. 
cual el artista se convierte en mediador, logrando transmitir en cada trazo del pincel su propio paisaje interior en consonancia con lo que recibe de la naturaleza»" La naturaleza representa el paradigma del pensamiento taoísta.

En el caso de Drury sus obras expresan esta idea, llegando a decir él mismo en varias ocasiones que no es él quien crea, sino que es la naturaleza la que habla. Los artistas salen al encuentro con la naturaleza, recolectan, modifican y comparten esa experiencia con el espectador, conviertiendo de ese modo su experiencia en arte. Este arte nos ofrece un lugar de reflexión en torno a la relación entre ser humano, arte y naturaleza. Su obra tiene por tanto un interés conceptual y trascendente más allá de su belleza. Estamos ante un arte que propone un reencuentro respetuoso del ser humano con el entorno natural y tiene una intención ecológica clara. Rasgos que también podemos apreciar en artistas como el escultor holandés Herman de Vries o el australiano John Davis, que dejan el paisaje en el que han actuado sin dańo alguno. Sin embargo, Drury tiene una relación íntima y espiritual con la naturaleza más evidente. Esta relación trascendente la apreciamos en el estudio de su obra $\mathrm{y}$ al leer las propias declaraciones del artista.

\section{CHRIS DRURY}

La cultura es el velo a través del cual describimos la naturaleza. La descripción del hombre de la naturaleza como algo separado, fuera de la ciudad, donde el límite es la división entre naturaleza y cultura, es una ilusión ${ }^{10}$.

Originario de Colombo, Sri Lanka, ha pasado la mayor parte de su vida en Inglaterra, donde vive. En la actualidad tiene 60 años. Para Drury el arte yace en las ideas, en el proceso de hacer o en los objetos creados que nos resultan sobrecogedores por su belleza. El método de trabajo es acudir a un lugar liberado de cualquier prejuicio, con una mente abierta, sin ninguna intención ni de captar su esencia conscientemente ni de alterar nada de su forma fundamental. Va con las mínimas expectativas posibles, sin imponer ninguna idea. Quiere sentir el lugar. En este aspecto, se ha visto influenciado por la doctrina del budismo zen y el tao: «Hacer arte, para mí, nunca es un medio de encontrar percepción. Es más bien el reflejo de una conciencia en crecimiento. Yo voy a la naturaleza 'externa', que es irreflexiva, vacía, para ver el todo. Del vacío viene la percepción que crea el arte». ${ }^{11}$ Como mencionábamos en el apartado anterior, esta relación espiritual y de clara influencia oriental es una seña de identidad de Drury que le diferencia claramente de otros artistas que practican al llamado «arte ambiental».

Su obra se centra en tres aspectos fundamentales: naturaleza y cultura, interior y exterior y microcosmos y macrocosmos. Para ello, colabora con científicos y

\footnotetext{
${ }^{9}$ Manrique, op. cit., 23.

${ }^{10}$ Chris Drury, Silent spaces (Escocia: Thames \& Hudson, 2004), 7.

${ }^{11}$ Chris Drury, Silence art espace with Kay Syrad (Escocia: Catleya, 2008), 12.
} 
técnicos de un amplio espectro de disciplinas y utiliza los medios visuales, las tecnologías y los materiales que mejor se adaptan a la situación. Desarrolla dos tipos de obras: por un lado, creaciones en lugares concretos que son generalmente colaboraciones con instituciones y pequeñas comunidades de todo el mundo. Estos trabajos pueden ser arquitectónicos (cámaras nubes), dibujos de la tierra, obras suspendidas, murales en la tierra, etc.; y, por otro lado, obras de menor formato, como objetos, vasijas, cestas, mapas... La mayoría de sus trabajos están realizados en piedra, estiércol, tierra, madera, hongos... y se desintegran, otros son desmontados por él mismo. "Como sucede con las pinturas tibetanas de arena, el objeto físico en el que se ha trabajado durante semanas desaparece, pero queda la esencia del trabajo en las mentes de las personas, la fuerza simbólica de ese foco de atención colectivo» ${ }^{12}$.

\section{COMIENZOS}

Drury empieza realizando esculturas figurativas hasta que conoce a Hamish Fulton a finales de los años 70. Los dos viajaron a Canadá para caminar por las montañas Rocosas. Esta experiencia le reveló una nueva forma de trabajo con la naturaleza muy diferente a su temprana escultura figurativa consistente en bustos, animales y pájaros. "Caminando por montańas, desiertos, bosques, en movimiento constante y frente a los elementos... percibía el interior y el exterior como una totalidad que fluye $»^{13}$. Las montañas le expandían fuera de sí mismo. A partir de esta experiencia empieza ver el mundo con amplitud de miras, no desde un punto de vista fijo.

Uno de sus últimos trabajos representacionales será Coyote Dream (figura 2), un animal tótem a través del cual mostraba el paisaje desértico de Texas. Sin embargo, a partir de 1982 toma la decisión de no realizar más trabajos de ese tipo y empieza a usar entonces el propio material del mundo. En ese mismo ańo crea la Medicine Wheel (figura 3). Recoge algo de la tierra cada día durante un ańo para crear un objeto-calendario. Está compuesta por 365 objetos naturales, ensartados entre los 24 radios de una rueda de dos metros y medio. Esto le lleva a vincular material y objeto a un momento localizado en tiempo y espacio, y a una fascinación por las plantas y sus propiedades, incluyendo los hongos, que están representados en el centro de la rueda. La rueda sigue el ritmo de las estaciones y el propio ritmo interno de la vida del artista, perpetuando en ella las alegrías y tragedias vividas en ese año, como el fallecimiento de su padre.

Según Drury, las plantas proporcionan nuestra primera conexión con el mundo exterior. Las plantas son la base de la cadena alimenticia, vinculándonos irreversiblemente con la tierra, y representan el arquetipo cultural de transformación de

12 Ibidem, 10.

${ }_{13}$ Hamish Fulton, The uncarved block: ten short walks in the Himalayas, 1975-2009 (Baden: Lars Müller Publishers, 2010), 23. 


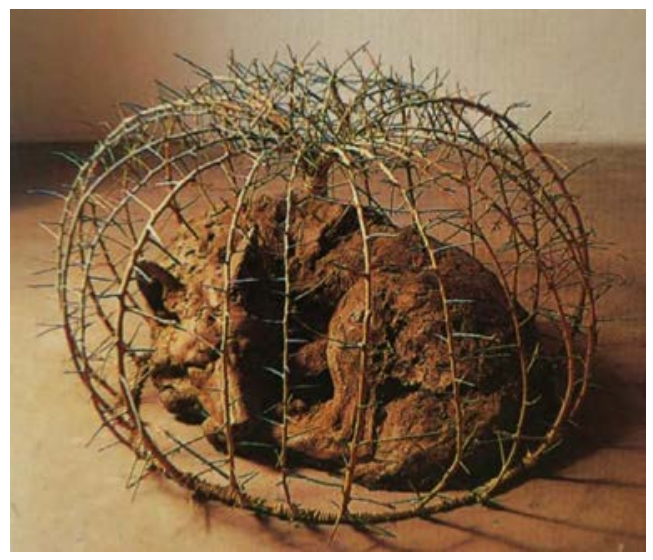

Figura 2. Coyote Dreams, 1980.

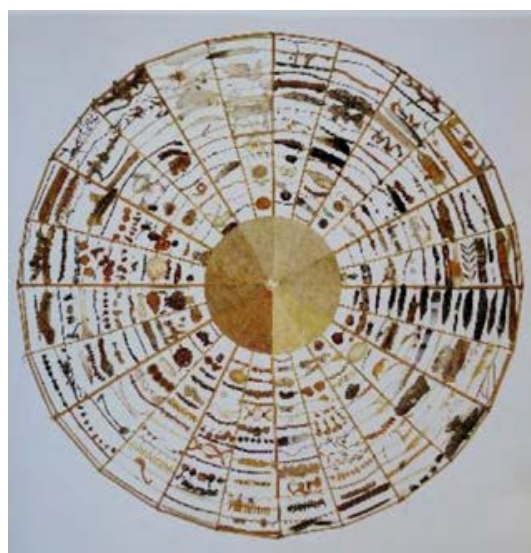

Figura 3. Cris Drury. Medicine Wheel 1982-1983. (C) Cris Drury.

la oscuridad a la luz, del submundo al cielo. La primera base de todo su trabajo -el movimiento entre fuera y dentro- es el componente oculto pero vital de esta obra.

Este trabajo contiene las semillas de todo lo que ha hecho desde entonces. Posteriormente descubrió que en la cultura nativo-americana se usaba una rueda medicinal, en una forma de cosmología, lo que le dio un significado cultural para el mundo exterior. No es la primera vez que le sucederá esto. A menudo crea obras y luego descubre que una forma igual ha sido realizada ya en el pasado. Esto no es sorprendente, teniendo en cuenta que su proceso es mayoritariamente intuitivo. En palabras del artista, es la naturaleza la que se comunica ${ }^{14}$. Los objetos mágicos son objetos que traslada ritualmente de un lugar a otro. Así, llevó a su casa un hueso de ballena que recogió en una playa y que revitalizó, marcó y frotó con tierra para convertirlo en una reliquia o talismán del lugar. A veces trenza los objetos. Son talismanes del tiempo y el espacio. Su existencia recuerda la experiencia vivida y la traslada al presente. El hecho de vincular objetos culturales con el mundo exterior (paisaje, lugar y tiempo) le inicia en un viaje de creación de refugios y cestas.

\section{REFUGIOS Y CESTAS}

El refugio es una necesidad básica humana. Drury trabaja con la idea de que el refugio tenga un interior y un exterior. «Me gusta cómo el espacio interior te lleva a tu propio interior, encerrándote, protegiéndote, del mismo modo que las montañas te sacan de ti mismo, impulsando tu mente y cuerpo más allá de sus lími- 

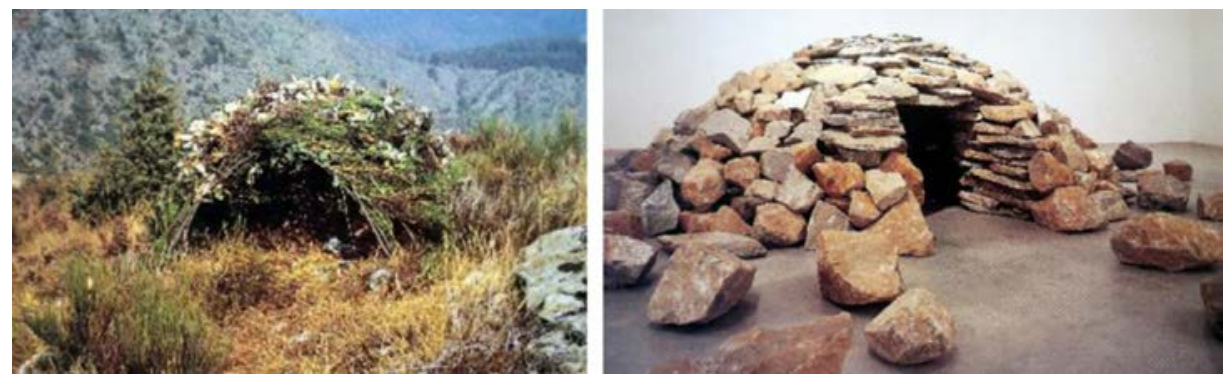

Figura 4. Shelter for herbars and healing, 1996, y Stone chamber, Los Ángeles, 1991. (C) Cris Drury.

tes normales» $»^{15}$. Algunos refugios los ha construido en un lugar particular porque era el momento de parar y montar el campamento para pasar la noche. Hay otros emplazamientos, normalmente cerca de donde vive, adonde acude específicamente para crear un trabajo. Son espacios que se pueden encontrar a la vuelta de la esquina de casas o pueblos. Construir allí requiere tranquilidad y cautela. Para aquellos que descubren las estructuras, son como hongos que han surgido durante la noche y con el tiempo se descompondrán en la tierra (figura 3). A veces los refugios son encargos, como Santuario del Desierto, construido en Irlanda con el artista Alfio Bonanno con el objetivo de crear un espacio donde estar y meditar ${ }^{16}$. Hay refugios construidos en interiores, como instalaciones en galerías, dominando el espacio (figura 4). Estos pasan de ser un refugio a ser un espacio cerrado, misterioso, fecundo. Realzan una vez más el intercambio dentro-fuera. Este intercambio se apreciará aún más en sus cámaras de nubes.

El círculo lo completan las cestas: según Gideon, en las culturas antiguas se creía que al hacer vasijas tejidas toda la psique de la tribu quedaba grabada en el tejido de la cesta ${ }^{17}$. Este sentido espiritual de los objetos fascina a Drury, ya que es una dimensión que nuestra cultura ha ido perdiendo. Para él realizar una cesta es un trabajo hipnótico, repetitivo, como una meditación, y como tal es transformador. Utiliza tierra, turba o arcilla. Algunas las recubre con estiércol convirtiéndolas en vasijas, unas para recordar viajes, otras para marcar momentos de euforia... Aquí la distinción entre arte y artesanía se rompe.

Según David Reason la creación de cestas puede centrar la mente, llevarla a percibir el verdadero orden del mundo. Transforma la ansiedad en una protec-

15 Ibidem, 20.

16 Ibidem, 24.

17 Sigfried Gideón, El presente eterno (Madrid: Alianza editorial,1995), 84. 

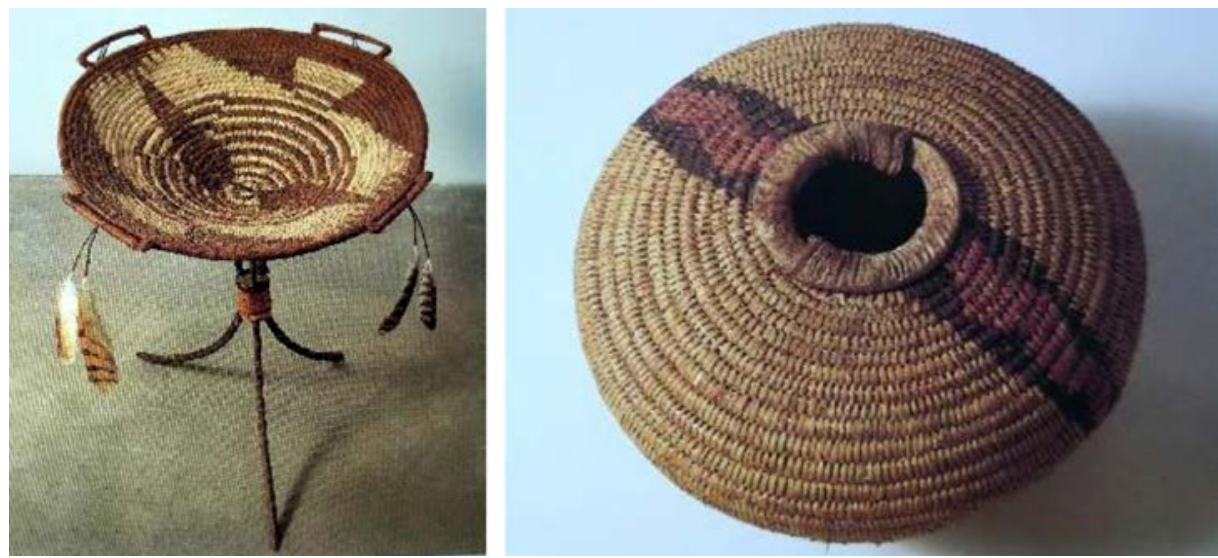

Figura 5. Dream Basket, 1985, y Basket for the moment between death and Life, 1985. (C) Cris Drury.

ción ambivalente ${ }^{18}$. Una cesta puede referirse a la naturaleza interior, al mundo de los sentidos, sueños e ideas. Drury tuvo un sueńo repetitivo que durante cuatro años permaneció en su mente hasta que pudo concretarlo en una cesta y descubrió que era un sueño arcaico. Así crea La cesta de los sueños (figura 5) respondiendo a la idea jungiana de haberse generado en el inconsciente colectivo ${ }^{19}$. La cesta para el momento entre la vida y la muerte (figura 4) la hizo para ese momento del año cuando el invierno está casi acabando pero la primavera aún no ha llegado; la tierra se pausa, como respirando hondo. Los cuernos en torno a la abertura de la cesta se unen el uno con el otro, representando la naturaleza cíclica del año. El diseño, el tejido y la naturaleza tediosa y repetitiva de este meticuloso trabajo tienen que ver con la mirada hacia el interior, del mismo modo que la actividad de pasear lo hace hacia el exterior.

En algunas ocasiones ha cogido una vasija tejida, la ha agrandado y le ha dado la vuelta para que actúe como refugio o como una válvula entre interior y exterior. Es el caso de las obras Mojón Cubierto, Domo del Cuco (figura 6) y Vortex: cesta a refugio, refugio a cesta.

En su análisis de la dialéctica entre naturaleza y cultura, Lucy Lippard observa que el arte podría ser parcialmente definido como una expresión de aquel momento de tensión cuando la intervención humana en, o en colaboración con,

${ }_{18}$ D. Reason, Landspace: Place, nature, material (Cambridge, Inglaterra: Kettle's Yard Gallery, 1986), 8.

19 Carl Gustav Jung, Arquetipos e inconsciente colectivo (Barcelona: Paidós, 2009). 


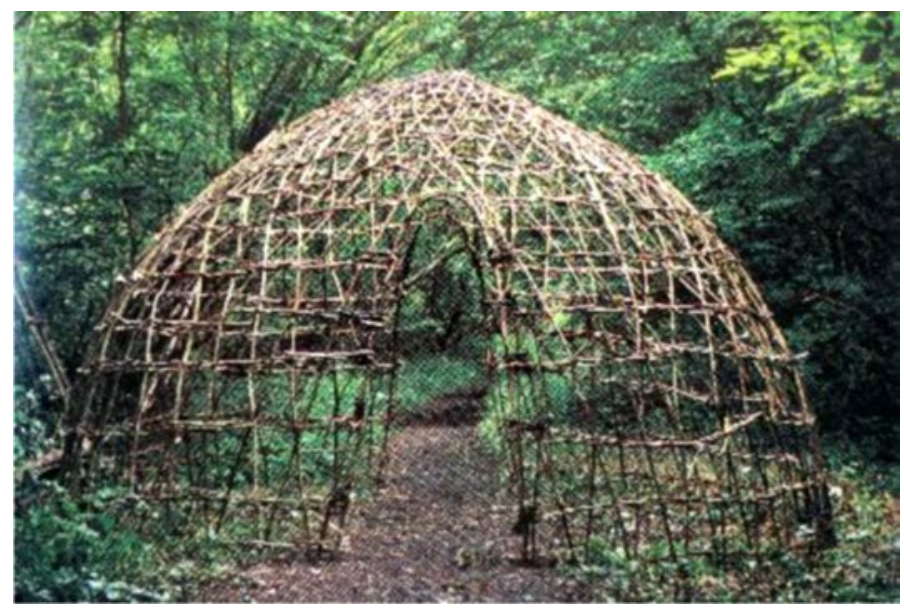

Figura 6. Cuckoo Dome, 1992. (C) Cris Drury.

la naturaleza se reconoce ${ }^{20}$. Drury va más allá de este reconocimiento para hacer de esa tensión el sujeto de su trabajo. Expone la dinámica entre naturaleza interna y externa, entre movimiento y quietud, entre proximidad y distancia. Transforma un refugio en una cesta, construye un refugio dentro de una galería, introduce un refugio de piedra dentro del tejido abierto de un domo a través del cual se ve una montańa, crea un remolino de piedras en el cauce de un río. Es precisamente en el momento en que estas tensiones se perciben cuando la relación del artista con la naturaleza se hace más transparente.

\section{MOJONES}

En Europa marcamos senderos y caminos con piedras. En otras partes del mundo los mojones tienen un significado religioso y pueden contener reliquias. Son señales que podemos identificar prácticamente en todas las culturas. Drury empieza a utilizar mojones para marcar un momento y un lugar en el tiempo. En contraposición al trasfondo meditativo de caminar, los mojones son señalizaciones de puntos culminantes, momentos de euforia a lo largo del camino. Un refugio es un lugar para parar, un espacio de descanso. Un mojón es una coma en el ritmo de un paseo. Los mojones de fuego son como una inspiración, una pausa mayor. Se puede tardar hasta una hora en construirlos y generan un contraste: fuego/agua, fuego/amanecer.

${ }^{20}$ Lucy R. Lippard, Seis años: La desmaterializacion del objeto artistico, de 1966 a 1972 (Madrid: Akal, 2004), 303. 
En Japón, tras finalizar un proyecto en el río Shimanto, en abril de 2004, decide, en la emoción del momento, poner cuatro pequeñas piedras del río una encima de la otra sobre una roca en una parte turbulenta del río. Tardó solo dos minutos y lo hizo gracias a la belleza del fluir del agua en ese lugar y al alivio de haber completado exitosamente su trabajo. El mojón era un momento de quietud entre el movimiento. A pesar de que era casi invisible entre las otras piedras, la mayoría de las personas que visitaron el lugar al día siguiente lo vieron y lo señalaron sonriendo al identificarse en todas las culturas. Según Drury, el horno de pan que construyó en St James, Piccadilly, en Londres en 1993, como un medio para que las personas sin hogar pudieran hervir agua en una tetera y estar calientes, era un reflejo de los mojones de fuego en lugares remotos. Al igual que los objetos trenzados, son talismanes del tiempo y el espacio ${ }^{21}$.

\section{TRABAJOS A GRAN ESCALA. LAS CÁMARAS DE NUBES}

Sus trabajos a gran escala son como recordatorios del paso del tiempo y de la presencia de otros pueblos antes de nosotros. En ellos una vez más está presente la idea de lo abierto y lo contenido, lo oculto y lo revelado ${ }^{22}$. Drury describe de una manera muy mágica su primera experiencia creando una cámara de nubes. La primera vez que probó la idea fue en el jardín de su pequeña ciudad, en Sussex (Inglaterra). Hizo un marco con palos doblados, los cubrió con plástico y lona, cortó una abertura de dos centímetros de diámetro en la parte superior y colocó una hoja de papel en el suelo. Era un día luminoso y soleado con ráfagas fuertes de viento que empujaban los cúmulos blancos de nubes a través del cielo. Dentro miró fijamente al papel, esperando a que sus ojos se ajustaran a la tenue luz. De repente el papel era azul y las asombrosas nubes blancas se estaban deslizando por él en silencio. Sacó sus pies y las nubes se deslizaron por sus zapatos. Era lo que esperaba, pero la belleza mágica y pura de esa imagen silenciosa le dejó fuertemente impresionado. Cada cámara oscura que ha creado desde entonces ha sido una variación de este primer refugio de lona ${ }^{23}$.

En las cámaras de nubes nos invita a percibir el mundo con la inocencia de un niño. Son muy simples y de tamaño humano. El incontenible exterior se introduce dentro de un espacio oscuro y contenido, permanente. Se encoge al tamaño del suelo o de una pared áspera y blanca de cal, en la que baila, mientras que las imágenes que vemos invertidas dentro están cambiando constantemente (figura 6). Le gusta la idea de que se pueda entrar en estas cámaras oscuras, terrosas (a veces bajo tierra), y en este espacio tranquilo ver qué se introduce del exterior y se invierte. Es

21 Drury, op. cit., 58.

22 A.M. Wolfe, Chris Drury: Mushrooms-Clouds (Chicago: Center for American places. Columbia University Chicago and The Nevada Museum Art, 2010), 5.

23 Drury, op. cit., 98. 

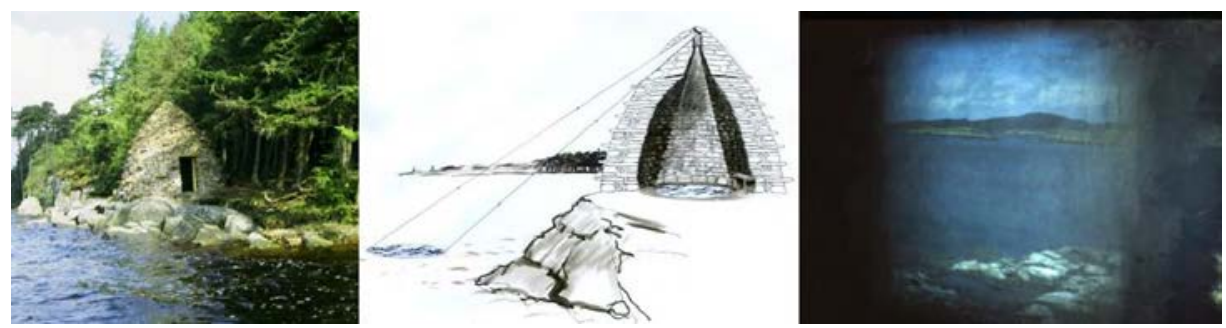

Figura 7. Wave Chamber. Kielder Reservoir. Nortumberland, 1996. (C) Cris Drury.

como una metáfora del movimiento de la oscuridad a la luz en los seres humanos. En algunas utiliza un periscopio de acero con un espejo y un cristal colocado en lo más alto para poder proyectar una imagen de las olas en el suelo interior (figura 7). La cámara construida en los jardines de St James, Picadilly, uno de los lugares más ruidosos de Londres, fue hecha con una estructura trenzada de avellano, revestido de piedra. Era un refugio temporal de arenisca, fácilmente desmontable. Estos jardines son un oasis de paz dentro de una ciudad caótica. Son utilizados por vagabundos como refugios y por trabajadores a la hora de la comida. El trabajo aquí era una declaración práctica y política, así como el mojón de fuego/horno de pan que lo acompañaba (mencionado en el apartado «Mojones»), y también ofrecía un foco de calma consciente en el ajetreo inconsciente de la vida diaria de la ciudad. Una ilusión de calma, ya que un espacio tranquilo y meditativo no garantiza una mente en calma. En la vida todo es movimiento, solo la mente tiene la posibilidad de estar en calma. Dentro de la quietud que ofrece la cámara hay una visión de movimiento en constante cambio. La idea que actúa de fondo en sus cámaras es esta: la naturaleza observada desde el refugio de la cultura.

El arte de Chris Drury es una historia de ritmos, tensiones y conflictos, trabajo, ritual e inteligencia humana. Cada obra es una narrativa completa, abriéndose gradualmente y alcanzando temporalmente una resolución. Cada decisión sobre qué material utilizar, qué forma crear, dónde situar el trabajo, decidir si grabarlo o no y las contradicciones entre el trabajo y su reproducción suponen un acto dramático que define, disuelve y renueva las percepciones sobre la relación entre naturaleza y cultura. Cuando viaja a algún sitio determinado para realizar un encargo implica a gente del lugar para que trabaje con él: artesanos, niños, artistas. La nacionalidad es algo sin importancia, lo que queda es el recuerdo del momento, la energía de realizar la obra. Maderuelo explica cómo la impermanencia de la obra en sí refuerza la dimensión espiritual del trabajo colaborativo ${ }^{24}$. Ha construido cámaras de nubes por

${ }_{24}$ Javier Maderuelo, La idea del espacio en la arquitectura y el arte contemporáneos 19601989 (Madrid: Akal, 2008), 66. 


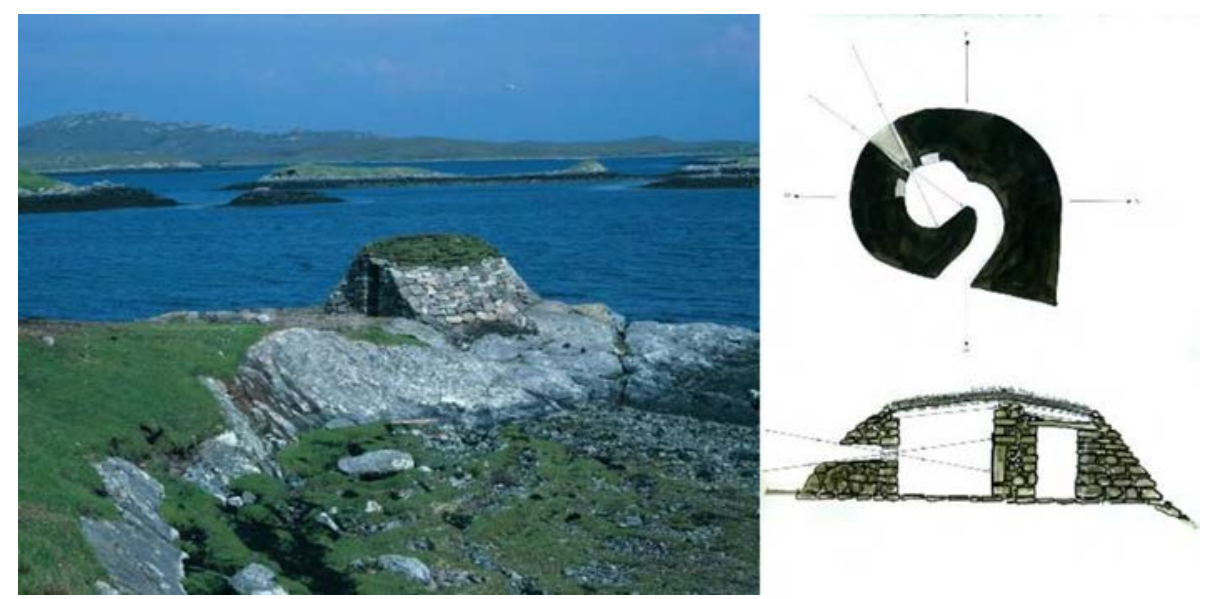

Figura 8. Hut of the shadow / Both nam faileas. Lochmaddy.

North Uist. Western Isles, 1997. (C) Cris Drury.

todo el mundo, aunque son especialmente reconfortantes las realizadas en pequeñas comunidades como Kielder, en Northumberland; Okawa-mura, en Japón; o Lochmaddy, en la isla escocesa de North Uist (figura 8). Es una tarea descomunal, pero que se disfruta mucho, ya que mucha gente se involucra. «Se comparten habilidades, y el tiempo y el trabajo generan amistades y risas gratuitas ${ }^{25}$.

\section{MACROCOSMOS, MICROCOSMOS Y MAPAS HUMANOS}

La teoría de la complejidad enuncia que a medida que un sistema se va volviendo más complejo, en lugar de degenerar en caos, tiende a formar patrones coherentes. Los sistemas de vida fluyen de una manera dinámica, en constante cambio, pero los patrones de relaciones interconectadas son una constante, a pesar de que puedan cambiar y evolucionar. Este es el patrón de vida en la tierra y la base de las últimas obras de Drury.

La relación entre el macrocosmos y el microcosmos es también un tema fascinante para el artista. Lo que persigue es buscar esas conexiones entre la naturaleza y el ser humano. Una planta en el Ártico puede recordar a una planta en las Cairngorms de Escocia. Están relacionadas, pero con pequeñas adaptaciones. Lo mismo sucede con los animales y los seres humanos, incluso las propias culturas: son lo mismo, con pequeñas diferencias. Este hecho interesa mucho a Drury; por

25 Drury, op. cit., 118. 


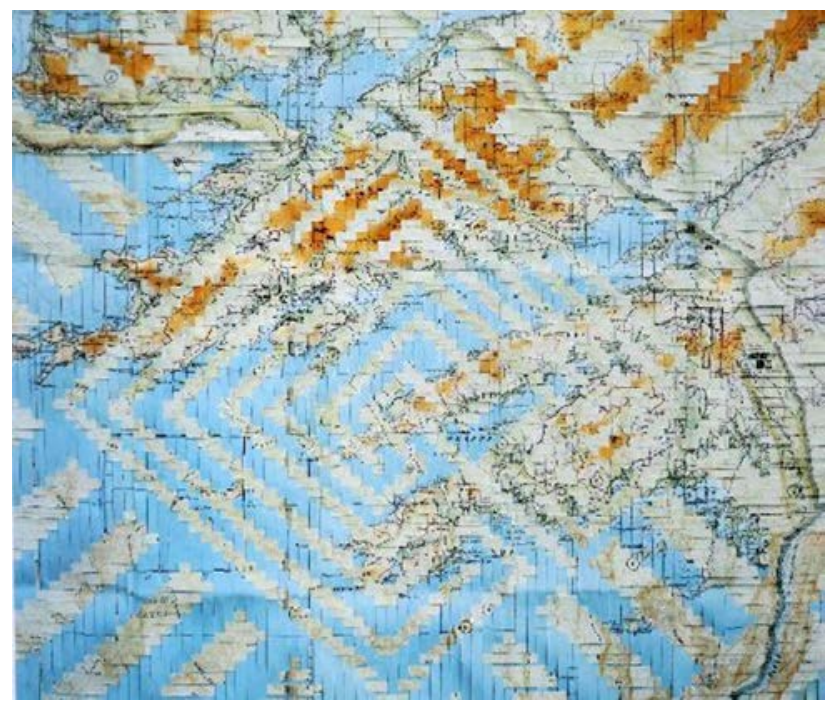

Figura 9. West cork / New México wowen maps, 1995. (CCris Drury.

ello entreteje islas, montañas, cordilleras y diversos lugares que ha visitado. Los trabajos de mapas trenzados son una extensión de la creación de cestas. En ellos muestra el mundo como una red tejida de interconexiones. Al cortar los mapas en tiras y entrelazarlas, a menudo une lugares opuestos: Las Hébridas/Manhattan o West Cork/Nuevo Méjico (figura 9). En relación con este último cabe señalar que la técnica del tramado se encuentra tanto en la cultura céltica como en la de los indios pueblo $^{26}$. En ambas obras ha unido similitudes físicas y culturales, convirtiéndolos en un todo. Las diferencias señalan aquello que hace nuestro mundo sorprendentemente bonito. La homogeneidad nos convierte en un todo.

\section{CONEXIONES CORPORALES Y NATURALEZA}

La presencia del agua y nuestra relación con ella es un hecho que se puede percibir a muchos niveles. El recogimiento y expansión de las mareas por los ciclos lunares diarios y anuales se refleja en ciclos dentro de nuestros propios cuerpos. Caminar durante días por la naturaleza, sobreviviendo en condiciones extremas climáticas, proporciona una experiencia corporal en la que nos hacemos conscientes del ritmo de nuestra respiración, la presión sanguínea en las venas y el efecto de la

26 A.M. Wolfe, Chris Drury: Mushrooms-Clouds, op. cit., 46. 

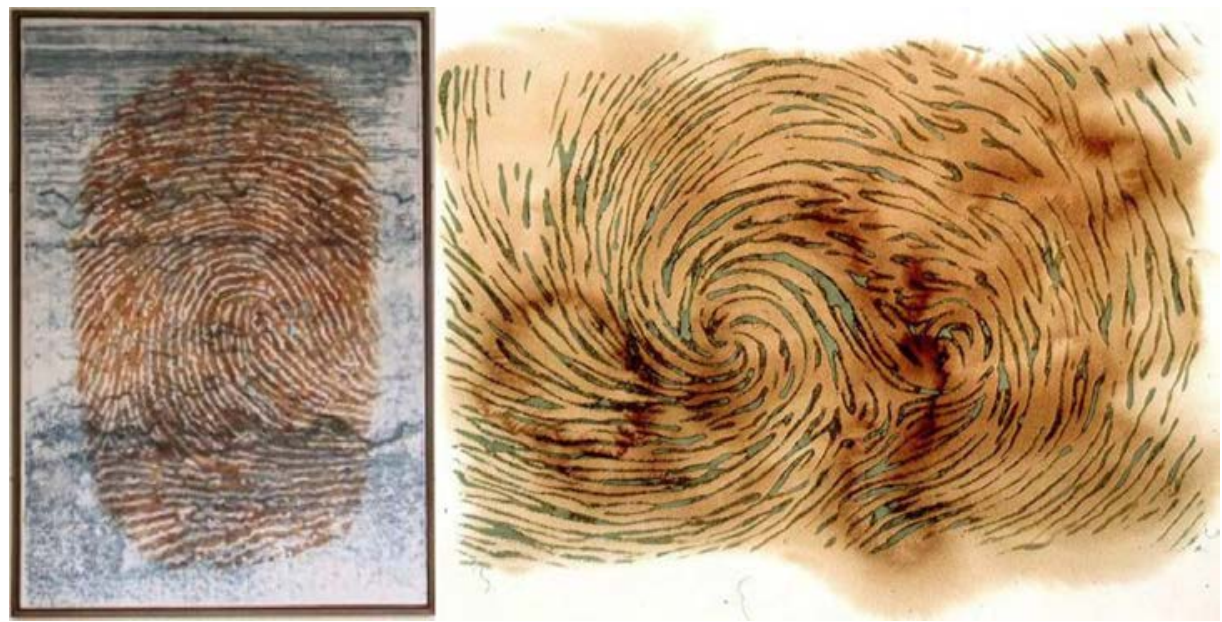

Figura 10. Heart River. Blood and river mud on paper, 1999, y

Hand on Heart. Mono printed with blood, 2002. (C) Cris Drury.

altitud en la cabeza. Estas conexiones naturales con los ritmos del cuerpo son muy profundas, incluso el óxido, usado por los pueblos indígenas como un pigmento sagrado, está presente en los glóbulos de nuestra sangre. En todo ello se basan las obras creadas por Drury que conectan el movimiento del agua y el cuerpo. Siendo nosotros parte de la naturaleza, no es sorprendente que la cultura siga los mismos patrones que la naturaleza. Patrones coherentes de forma, cambio y reforma. En ellas se apunta que el caos puede ser simplemente la incapacidad de ver esas conexiones. Río-corazón, por ejemplo, es un diseño del patrón de remolino de agua en sangre y barro. Su propia sangre derramada en papel húmedo y absorbida por él. Después trazó un dibujo de las fibras espirales en el ápice del corazón humano pintado con barro de río. El corazón está formado por la forma en la que la sangre fluye, es lo mismo para los ríos y el agua: los tejidos del corazón están determinados por los patrones del fluir de la sangre de la misma manera que los ríos forman cauces serpenteantes en la tierra ${ }^{27}$. Trabaja con ecocardiogramas del corazón entrelazados con mapas de los Pirineos. Los patrones de ondas recuerdan a las pinturas chinas de paisajes montańosos: cuanto peor es el problema cardiaco, más accidentado es el terreno. Las huellas dactilares aparecen también en este tipo de obras. De la misma forma que las ondas y espirales en la madera de un árbol siguen los movimientos del flujo de la savia, las huellas dactilares son un indicador del movimiento de los fluidos dentro del cuerpo (figura 10).

27 Drury, Silence art espace op. cit., 8. 


\section{REFLEXIONES Y APORTACIONES FINALES}

La finalidad del presente estudio era recorrer la obra de Chris Drury desde el punto de vista del artista y ver su relación con el arte, la naturaleza y la trascendencia. Como vía fundamental para lograr este objetivo, hemos realizado un recorrido por las principales etapas de su obra y sus intenciones más íntimas al realizarlas. Hemos podido constatar que forma parte de estos artistas atemporales que participan del pensamiento filosófico taoísta, y que propone la integración entre la naturaleza y el ser humano como única posibilidad de mantenernos en armonía con el universo. Jackson Pollock una vez dijo: «Soy Naturaleza... Trabajo de adentro hacia afuera, como la naturaleza ${ }^{28}$. Drury empieza desde una posición diferente, para él imaginar que trabaja de dentro hacia fuera, o desde fuera hacia dentro, supondría interrumpir la dinámica entre estas dos posibilidades que, como hemos observado en sus obras, utiliza indistintamente. El ser es como una puerta que oscila entre dentro y fuera, que permite un flujo libre del uno al otro. A través de sus obras, nos invita a reflexionar sobre la forma en que miramos todo, sin pararnos realmente a «ver».

La conclusión a la que nos lleva con sus cámaras de nubes es que miramos la realidad desde el refugio de la cultura. Con sus ecocardiogramas se propone entender el movimiento eterno de la naturaleza para poder observar nuestros propios ciclos efímeros y ahondar en esas conexiones que nos devuelven el vínculo con nuestro lado más trascendente. Para sentirnos uno con lo que nos rodea. Al compararlo con otros artistas del Land Art hemos podido constatar cómo Drury tiene una postura integradora que propone la observación sensible e intuitiva de nuestro entorno y de las influencias que este ejerce sobre nosotros para aprender de esa experiencia. Sutilmente apunta que tal vez nuestro caos proviene de la incapacidad de ver estas conexiones.

Nuestra principal contribución es hacer más visible a un artista como Cris Drury fuera de su contexto habitual. Artista que transmite en cada fragmento de su obra su propio paisaje interior y actúa en consonancia con lo que recibe de la naturaleza. Somos conscientes del número de artistas pendientes por añadir al estudio y reivindicamos por tanto la contribución de estos al arte contemporáneo. Para concluir ańadimos una última reflexión al respecto: la contemplación implica un estado íntimo de serenidad, durante el cual la actividad mental disminuye para dejar una mayor dimensión a los sentidos y rescatarlos del pensamiento superfluo, de la actitud analítica, de la mirada insustancial. En la contemplación, la naturaleza se manifiesta intuitivamente a través de la mirada interior y se abre paso a la percepción. La realidad se nos revela como parte de nuestro propio ser, una revelación que nos llevará a comprender que, al igual que cualquier otra manifestación del universo, los seres humanos somos naturaleza, tanto en su diversidad como en su integridad. En palabras del propio Drury:

${ }^{28}$ Manrique, op. cit., 18-22. 
Un artista es un comunicador, pero para ser artista uno tiene que ser primero un ser humano, completo si es posible. Desde esta posición no hay división entre hombre, arte y naturaleza. El mundo es percibido tal y como es. Personalmente no tengo nada que comunicar, el trabajo simplemente refleja el movimiento entre momento y momento en el mundo, tal y como es, por tanto, es la naturaleza misma la que comunica ${ }^{29}$.

Recibido: mayo de 2019; ACEPTADo: noviembre de 2019

29 Drury, op. cit., 6. 


\section{BIBLIOGRAFÍA}

Drury, Chris. 2004. Silent spaces. Londres: Thames \& Hudson.

Drury, Chris. 2008. Silence art espace with Kay Syrad. Escocia: Catleya Editions.

Fulton, Hamish. 2010. The uncarved block ten short walks in the Himalayas, 1975-2009. Baden: Lars Müller Publishers.

Gideón, Sigfried. 1995. El presente eterno. Madrid: Alianza editorial.

Gooding, Mel. 2017. Song of the earth. European artist and the landscape. Londres: Thames \& Hudson.

Jung, Carl Gustav. 2009. Arquetipos e inconsciente colectivo: Barcelona: Paidós.

LaILACH, Michael. 2007. Land art. Colonia: Taschen.

Lippard, Lucy R. 2004. Seis años: La desmaterializacion del objeto artistico, de 1966 a 1972. Madrid: Akal.

Maderuelo, Javier. 2008. La idea del espacio en la arquitectura y el arte contemporáneos 1960-1989. Madrid: Akal.

Manrique, María Eugenia. 2018. Arte, Naturaleza y espiritualidad. Barcelona: Kairós.

Malpas, William. 2016. The art of Andy Goldsworthy. Maidstone, Kent: Crescent Moon Publishing.

Raquejo, Tonia. 1998. Land Art. San Sebastián: Nerea.

Reason, D. 1986. Landspace: Place, nature, material. England: Kettle’s Yard Gallery.

Talens Pardo, Anna. 2011. La transformación de la experiencia de la naturaleza en arte. El nomadismo y lo efímero. Tesis doctoral. Universitat Politècnica de València.

Wolfe, Anne M. 2010. Chris Drury: Mushrooms-Clouds. Chicago: Center for American places. Columbia University Chicago and The Nevada Museum Art. 